\title{
Neurodevelopmental outcomes in paediatric immune-mediated and autoimmune epileptic encephalopathy
}

Sukhvir K Wright ${ }^{1,2}$ and Amanda G Wood ${ }^{1,3}$

${ }^{1}$ School of Life and Health Sciences \& Aston Neuroscience Institute, Aston University, Birmingham, UK

${ }^{2}$ Department of Neurology, Birmingham Children's Hospital, Birmingham, UK

${ }^{2}$ School of Psychology, Faculty of Health, Melbourne Burwood Campus, Deakin University, Geelong, Victoria, Australia 


\begin{abstract}
$\underline{\text { Abstract }}$
Recognition of paediatric autoimmune/ immune-mediated encephalitis and epileptic encephalopathy (e.g. NMDAR-Ab encephalitis) has rapidly increased over the last ten years. While we are succeeding in the diagnosis and identification and even early treatment of these encephalitidies, with studies describing $>80 \%$ are making a "good" recovery, we are now recognising that a "good" medical outcome does not cover the cognitive, social and behavioural sequelae that can occur, particularly in paediatric patients. Basic measures of medical outcome, for example the modified Rankin Scale (MRS) or the Paediatric Cerebral Performance Category (PCPC), offer the advantage of being quick to use, but do not reveal the more complex difficulties that can impact the future of affected children. This article reviews the current literature on neurodevelopmental outcomes in children affected with autoimmune and immune-mediated encephalitis/ epileptic encephalopathy and provides guidance on post-onset surveillance aimed at identifying those most likely to experience ongoing long-term difficulties.

Acknowledgements

AW is supported by a European Research Council Consolidator Fellowship (ERC-CoG2015PROBIt-682734). SW was funded by an Epilepsy Research UK Postdoctoral Fellowship (F1601) and a Wellcome Trust Clinical Research Career Development Fellowship (216613/Z/19/Z) during the course of this work.
\end{abstract}




\section{$\underline{\text { Introduction }}$}

Immune-mediated/ autoimmune epileptic encephalopathy (AEE) is characterized by the presence of neuronal autoantibodies that bind to proteins essential for normal brain function and development. When synaptic proteins are targeted (e.g. the NMDA receptor), paediatric patients present with a range of neurological and neuropsychiatric symptoms including seizures, movement disorder, and behavioural change. Acute demyelinating events (e.g. optic neuritis, transverse myelitis) occur with encephalopathy and seizures when autoantibodies are directed against myelin associated proteins, i.e. myelin oligodendrocyte (MOG). Regardless of their differing autoantibody targets, paediatric AEEs all have the potential to cause long-term adverse neurodevelopmental changes as well as the severe acute neurological and neuropsychiatric syndromes. This review briefly summarises the diagnosis and treatment of the common peadiatric AEEs and then focuses on current knowledge regarding neurodevelopmental outcomes.

\section{N-methyl-D-aspartate receptor antibody (NMDAR-Ab) encephalitis}

NMDAR-Ab encephalitis was first described in 2007 as a paraneoplastic neuroimmune disorder primarily affecting young women with ovarian teratoma ${ }^{1}$. It is now widely recognised as the most common AEE, and has been described in patients of all ages, with and without the presence of tumours. Affected patients present with seizures, neuropsychiatric symptoms, cognitive and autonomic dysfunction, movement disorder and decreased level of consciousness ${ }^{2}$. In children, seizures, abnormal movements, insomnia, and irritability are the more frequent symptoms recognised, whereas in adults, abnormal behavior and psychosis are more likely to herald the onset of disease ${ }^{3}$. In pre-school children, abnormal behavior may initially be misdiagnosed as another neurodevelopmental disorder such as autism ${ }^{4}$. Estimated incidence in UK children is 0.85 per million children per year ${ }^{5}$, and paediatric cases make up over a third of reported cases in the literature ${ }^{3}$. Diagnosis is made on the basis of clinical features and the detection of NMDARantibodies directed against the NRI subunit in the cerebrospinal fluid (+/- serum) of affected patients ${ }^{6}$. The EEG is almost always abnormal showing focal or diffuse slowing/encephalopathy, epileptic activity, or extreme delta brush ${ }^{3,7,8}$. Brain MRI is only reported as abnormal in approximately $33 \%$ of patients, and not associated with clinical severity or neurological outcome

3. However, this disparity is explained by functional MRI and connectivity studies which do 
demonstrate abnormalities in hippocampal and frontoparietal network connectivity despite the majority having unremarkable routine brain MRI ${ }^{9}$.

The standard treatment regime for paediatric AEE including NMDAR-Ab encephalitis begins with first-line immunotherapy (e.g. steroids, immunoglobulins or plasma exchange), escalating rapidly to second line agents if there is no sign of clinical improvement (e.g. rituximab, cyclophosphamide). Although early use of rituximab in paediatric CNS autoimmune and inflammatory diseases is associated with improved outcomes ${ }^{10}$, no randomized controlled trial evidence currently exists that compares the stepwise immunotherapy approach to initiation of rituximab from day of diagnosis.

Recovery and outcomes in paediatric NMDAR-Ab encephalitis are measured primarily through basic assessment criteria, for example, the five point modified Rankin scale (mRS) ${ }^{3,5,7}$. With early recognition and prompt treatment, a significant neurological improvement is seen in up to $85 \%$ of paediatric patients using theses scales ${ }^{3,5}$. More recently, however, smaller retrospective paediatric studies with more detailed functional and neuropsychological outcomes have been published and rather worryingly, suggest that compared to adults, children with NMDAR$\mathrm{Ab}$ encephalitis may have long-term effects impacting daily life ${ }^{11}$. Using the Adaptive Behaviour Assessment System (ABAS-3), this study showed that pediatric patients had changes in adaptive behaviour that were not captured when focusing on neurological disability using the mRS scale. The differences in adaptive behavior observed suggested that children may be left with long-term impairment following NMDAR-Ab encephalitis, whereas adults assessed in a comparable way appeared to regain normal function. Subtle neurocognitive deficits may have affected the adaptive behavior resulting in an increased likelihood for affected children to require additional support at home and/or school for everyday living. Data from a larger retrospective study of 28 paediatric patients seen at a median of 31 months after disease, found that many patients had cognitive problems and fatigue, and this was associated with lower academic achievement and poorer quality of life ${ }^{12}$. Lower scores were recorded in the sustained attention, speed and short -term verbal memory and language domains. Surprisingly, important predictors for "good" mRS outcomes (e.g. early treatment) were not associated with neuropsychological outcome, again emphasising the notion that $\mathrm{mRS}$ scales do not reflect the complete picture of recovery. One recent prospective study of 10 children found deficits in all domains tested in the acute phase of illness (general 
intellectual abilities, receptive and expressive language, short-term verbal memory, planning, selective and sustained attention and visual-motor integrations $)^{13}$. During subsequent follow-up, general intellectual abilities were within normal limits at the most recent assessment (median 31 months). Nevertheless, specific cognitive deficits were still present in over half of the patients at the same time-point, mainly affecting verbal fluency, working memory, executive functions and short-term memory. These persisting deficits affected quality of life, social relationships and academic achievement, but despite this, all children resumed their everyday lives ${ }^{13}$. A small series of six children (6 to 13 years at first assessment) tested within one month of discharge through to 24 or more months also highlighted the presence of early impairments in executive skills and information processing speed that persisted at long-term follow up in up to half of cases ${ }^{14}$. Visual motor functions were also impaired and in a seventh case assessed first at 18 months of age, deficits emerged over the course of two years. Most longitudinal studies do show improvement in cognitive processing over time which is encouraging for patients and families. However, the concern is that while patients report that their children have no residual difficulties, cognitive impairments that impact on social and academic functioning are still detected on formal testing ${ }^{15}$. It is unclear whether this represents true deterioration, perhaps reflecting late-effects of the incident neurological event or its treatment, or rather a failure to attain developmentally appropriate skills in line with peers. The latter phenomenon is well recognized in the paediatric neuropsychology literature and this "early vulnerability" 16 highlights the need to understand the specific profile of outcomes in children with AEE. This in turn underscores the importance of clinicians being aware of the long-term persistence of neuropsychological deficits in children following NMDAR-Ab encephalitis, in order to counsel families and recommend access to neuropsychology testing. Given the relative paucity of high quality studies in the area, it is difficult to recommend a comprehensive set of assessments for this cohort of children. However, based on existing findings, referral for age-appropriate measurement of general intellectual abilities, new learning, executive functions and information processing speed seems warranted. Furthermore, screening for behavioural impairments, again with age appropriate measures of symptomatology, is appropriate. The timing of assessments should be planned for key transition points in the young person's education (into primary school, transition to secondary and/or further education).

Ideally, early recognition of neuropsychological deficits will allow timely, specific interventions aimed to improve long-term neurodevelopmental outcomes and support families of children with 
impairments to receive tailored input from school and allied health services. Further prospective longitudinal studies are required to identify which children are likely to experience neurodevelopmental sequelae, as well as the effect of treatment on subsequent brain development.

The other autoantibodies associated with childhood AEE along with available outcome data are summarized in Table 1 below.

\begin{tabular}{|c|c|}
\hline $\begin{array}{l}\text { Neuronal } \\
\text { target }\end{array}$ & Clinical features and pediatric outcome data \\
\hline $\begin{array}{l}\mathrm{GABA}_{A} \\
\text { receptors }\end{array}$ & $\begin{array}{l}\text { Severe autoimmune encephalitis with refractory seizures and extensive MRI cortical/subcortical } \\
\text { FLAIR abnormalities }{ }^{17,18} \text {. Antibodies bind to co-expressed } \alpha 1 / \beta 3 \text { or } \alpha 1, \mathrm{~V} 2 \text { subunits }{ }^{18,19} \text {. Strong } \\
\text { association with underlying tumour in adults. } \\
\text { Most patients, including children, present with refractory status epilepticus or seizures, as well } \\
\text { as varying symptoms of memory, cognitive and affective problems, and movement disorder }{ }^{17} \text {. } \\
\text { Only one child made a complete recovery in this series of } 10 \text { cases ( } 8 / 10 \text { partial recovery; } 1 / 10 \\
\text { death) }{ }^{18} \text {. } \\
\text { Cases also described of Febrile Infection-Related Epilepsy Syndrome (FIRES), a devastating } \\
\text { epilepsy of childhood } 20 \text {. Early treatment with immunotherapy is associated with improved } \\
\text { outcomes in children }{ }^{21,22} \text {. }\end{array}$ \\
\hline LGI1 & $\begin{array}{l}\text { The Leucine-rich glioma inactivated } 1 \text { (LGI1) protein is complexed to the voltage gated } \\
\text { potassium channel (VGKC-complex) which functions to control membrane excitability. Found in } \\
\text { adults with facio-brachial dystonic seizures, which can evolve into limbic encephalitis (LE) } \\
\text { without prompt recognition and treatment }{ }^{23} \text {. } \\
\text { One case report of } 14 \text { year old boy with Type } 1 \text { diabetes mellitus presenting with subacute } \\
\text { memory dysfunction, left hippocampal swelling on imaging, and positive oligoclonal bands in } \\
\text { the cerebrospinal fluid. Good recovery with plasma exchange and immunotherapy, although } \\
\text { residual memory problems remained }{ }^{24} \text {. }\end{array}$ \\
\hline $\begin{array}{l}\mathrm{GABA}_{\mathrm{B}} \\
\text { receptor }\end{array}$ & $\begin{array}{l}\text { Seizure predominant limbic encephalitis phenotype, associated with small-cell lung carcinoma } \\
\text { in adults, very rare in children }{ }^{25} \text {. Single paediatric case, aged } 3 \text { years, presented with mixed } \\
\text { movement disorder and refractory seizures, died of overwhelming sepsis despite } \\
\text { immunomodulatory therapy }{ }^{26} \text {. }\end{array}$ \\
\hline $\begin{array}{l}\text { AMPA } \\
\text { receptor }\end{array}$ & $\begin{array}{l}\text { Limbic encephalitis phenotype, rare in adults and children. One } 14 \text { year old patient described } \\
\text { with favourable outcome post immunotherapy (mRS of } 1)^{27} \text {. Two cases of childhood onset } \\
\text { Rasmussen encephalitis (RE) positive for AMPAR-Abs, likely to be secondary to inflammatory } \\
\text { changes rather than causative; both had a very typical course of } \mathrm{RE}^{28} \text {. }\end{array}$ \\
\hline $\begin{array}{l}\text { Glycine } \\
\text { receptor }\end{array}$ & $\begin{array}{l}\text { Most commonly associated with Progressive encephalomyelitis with rigidity and myoclonus in } \\
\text { adults (PERM) })^{29} \text {. Can present with epileptic encephalopathy, rare in children ( } 3 \text { paediatric } \\
\text { cases in literature, } 2 / 3 \text { responded well to immunotherapy }{ }^{30} \text { ). }\end{array}$ \\
\hline GAD65 & $\begin{array}{l}\text { Intracellular antigen. Paraneoplastic antibody, mainly associate with limbic encephalitis and } \\
\text { stiff-person-syndrome in adults }{ }^{31} \text {. Rarely reported in children, invariably associated with poor } \\
\text { outcome. }{ }^{32}\end{array}$ \\
\hline
\end{tabular}

Table 1. Neuronal targets and outcomes in paediatric autoimmune epileptic encephalopathies 
Acute Disseminated Encephalomyelitis (ADEM) and Myelin oligodendrocyte antibodies (MOG$\underline{\text { Abs) }}$

Generalised or focal seizures are frequently seen in the presentation of Acute Disseminated Encephalomyelitis (ADEM), a CNS inflammatory demyelinating disease of childhood, characterised by encephalopathy, polyfocal CNS deficits and multifocal brain MRI lesions ${ }^{33-35}$. Response to immunotherapy is good, and outcomes generally favourable, however, some patients may relapse ${ }^{33}$. Whilst most children with ADEM go on to develop in line with their peers, up to $43 \%$ show impairments in specific cognitive or behavioural domains. Although meta-analysis did not reveal overall differences, it seems that a subset of children appears to have ongoing cognitive (e.g. processing speed) or behavioural difficulties (internalizing symptoms) ${ }^{36}$. Furthermore, earlier work raised concern that children with early onset ( $<5$ years) were more likely to have significant social and cognitive impairments at follow up ${ }^{37}$.

Antibodies to the myelin-oligodendrocyte protein (MOG-Abs) have been identified in ADEM patients, and appear to predict a non-multiple sclerosis but multiphasic disease course ${ }^{38,39}$. MOG$\mathrm{ab}$ associated disease includes adult cases of unilateral cerebral cortical encephalitis presenting with generalised epileptic seizures, with or without encephalopathy, which are responsive to antiepileptic drugs and immunotherapy ${ }^{40}$. This highlights that MOG-Ab associated disease goes beyond white matter effects, with patients more likely to present with seizures as well as encephalopathy. With the relapsing nature of the disease spectrum associated with MOG-Abs, as well as the encephalitis and grey matter effects, there is concern that without early identification and treatment, there may be long lasting cognitive sequelae. This is of particular concern in paediatrics as there is a predilection for brain lesions in the younger age group $(<9 \mathrm{yrs}){ }^{27}$. Recent cohort studies in both children and adults show that MOG-Ab associated disease is more likely to present with seizures, and incur an increased risk of developing long-term "autoimmune epilepsy" 41, 42. A recent UK study in MOG-Ab positive ADEM paediatric patients showed a trend towards a greater risk of post ADEM epilepsy associated with MOG-Abs, which may be the result of ongoing subclinical inflammation. This hypothesis was supported by the higher rate of intrathecal oligoclonal bands detected in these patients ${ }^{42}$. MOG-Ab associated disease may reflect a true antibody-mediated epilepsy syndrome, and treatment may best be directed towards ameliorating the ongoing inflammation. Although studies show that $30 \%$ of children with MOG antibodies will 
relapse within 2 years ${ }^{43}$, at the moment we cannot predict which children will relapse or recover, and we do not know how MOG-Abs directly contribute to the problems seen. Long-term cognitive problems are seen in up to $50 \%$ of children with MOG-Ab disease and brain involvement, but there are marked differences in both the progression of cognitive impairment and rate of accumulation of physical disability ${ }^{44,45}$.

In ADEM, rehabilitation is focused on the motor impairment during the acute phase. Presentation with cognitive impairment can be late, and therefore the window of cognitive rehabilitation is often missed. Most studies make use of the EDSS (Extended Disability Severity Score) to measure outcome which is more representative of physical disabilities as opposed to cognitive problems, so underreporting of cognitive sequelae is a possibility. With the uncertainty surrounding whether or not a child with MOG-Abs will relapse, treatment and prognostication of these antibody positive cases is particularly challenging ${ }^{39}$.

\section{Future challenges}

One of main prognostic and treatment challenges in children with immune-mediated and autoimmune epileptic encephalopathy is identifying those most at risk of severe disease and longlasting cognitive dysfunction when they first present. There is a pressing need to invest in collaborative cohort studies to track the natural history of disease and recognize the potential predictive features of poor outcome to facilitate timely effective therapeutic interventions. Although some studies show that affected children may be at a higher risk of long-term impairment than adults, the exact relationship between age of disease onset and eventual outcome is far from clear. Confounding factors include the late recognition of disease in children if the presentation is not typical causing a delay in treatment onset. The NEOS (anti-NMDAR Encephalitis One-Year Functional Status) score was developed using retrospective data from 382 NMDAR-Ab encephalitis patients to score affected patients within 4 weeks of initiating treatment and predicts the probability of good functional status at 1 year after initial symptom presentation ${ }^{46}$. Treatment delay of more than 4 weeks, absence of improvement within 4 weeks of starting treatment, abnormal MRI, and elevated CSF white blood count were independent predictors for outcome and each assigned one point to construct the score. Although the score correlated with the probability 
of poor functional outcome at 1 year, patients with high NEOS scores at outset still progressed to recovery after 1 year. Therefore, functional status at one year did not represent the final clinical outcome, but was useful in delineating the speed of clinical improvement ${ }^{46}$. The development of similar tools to predict ultimate clinical outcomes using prospective standardized patient datasets should be the aim of future trials. Consideration should also be given to including a qualitative component in long-term outcome studies, in order to capture patient and family perspectives at crucial time points during recovery ${ }^{47}$.

Immunotherapy is the mainstay of treatment for paediatric immune-mediated epileptic encephalopathy and aims to remove circulating neuronal autoantibodies or halt their production. Neuronal antibodies target ion channels and receptors (antigens), disrupting neuronal networks at a synaptic level during critical time points in a child's development. There are, as yet, no available treatments that ameliorate the specific synaptic effects. This could also be contributing to the longterm recovery of children with, for example, NMDAR-Ab mediated autoimmune epileptic encephalopathy. Pre-clinical studies in rodent models have shown promise in the use of agents that modulate the function of the NMDAR at the synapse ${ }^{48-50}$, the future challenge will be to translate these findings to the bedside. Adjunctive individualised therapies that rescue the target antigen dysfunction may allow reduction in the amount of immunotherapy required, thereby minimising potential side-effects, and prevention of long-term cognitive problems.

Finally, the increased use of advanced imaging and neurophysiology techniques may also help improve identification of underlying brain dysfunction and cognitive impairment early in the disease course. The use of resting state functional MRI and connectivity studies in NMDAR-Ab encephalitis ${ }^{9,51}$ show potential clinical utility, however translation to standard clinical practice is hampered by the small numbers and retrospective analysis. Another important tool for studying intrinsic brain activity is resting-state connectivity estimation with magnetoencephalography studies (MEG). Global connectivity analysis can highlight abnormal functional networks in different frequency bands. For example, in multiple sclerosis (MS) patients a lower functional connectivity in the alpha 2 band and higher functional connectivity in the beta band have been recently found ${ }^{52}$. More recently, using clinically applicable MEG-measures it has been demonstrated that there is a clinically relevant slowing of neuronal activity in MS patients in parietotemporal cortical areas and the thalamus, strongly related to cognitive impairment ${ }^{53}$. These 
findings illustrate the relationship between thalamic atrophy, altered functional connectivity and clinical and cognitive dysfunction in MS, which could serve as a bridge to understand how neurodegeneration is associated with altered functional connectivity and subsequently clinical and cognitive decline. The application of resting-state MEG as a biomarker for cognitive disturbances in MS and other demyelinating diseases such as MOG-Ab disease in a clinical setting is therefore an increasing possibility.

To date, no study has related the clinical, paraclinical, radiological and neurophysiological features at presentation to eventual neuropsychological and neurodevelopmental outcome in peadiatric patients with immune-mediated epileptic encephalopathy. This would enable early identification of children at risk, leading to better support and cognitive rehabilitation allowing children to reach their educational potential, later their vocational potential. Setting the expectations and maximising children's developmental trajectory will improve the quality of life for children and their families. 


\section{$\underline{\text { References }}$}

1. Dalmau J, Tuzun E, Wu HY, et al. Paraneoplastic anti-N-methyl-D-aspartate receptor encephalitis associated with ovarian teratoma. Annals of neurology 2007;61:25-36.

2. Dalmau J, Gleichman AJ, Hughes EG, et al. Anti-NMDA-receptor encephalitis: case series and analysis of the effects of antibodies. Lancet neurology 2008;7:1091-1098.

3. Titulaer MJ, McCracken L, Gabilondo I, et al. Treatment and prognostic factors for longterm outcome in patients with anti-NMDA receptor encephalitis: an observational cohort study. Lancet neurology 2013;12:157-165.

4. Hacohen Y, Wright S, Gadian J, et al. N-methyl-d-aspartate (NMDA) receptor antibodies encephalitis mimicking an autistic regression. Developmental medicine and child neurology 2016;58:1092-1094.

5. Wright S, Hacohen Y, Jacobson L, et al. N-methyl-D-aspartate receptor antibodymediated neurological disease: results of a UK-based surveillance study in children. Archives of disease in childhood 2015;100:521-526.

6. Graus F, Titulaer MJ, Balu R, et al. A clinical approach to diagnosis of autoimmune encephalitis. Lancet neurology 2016;15:391-404.

7. Armangue T, Titulaer MJ, Malaga I, et al. Pediatric anti-N-methyl-D-aspartate receptor encephalitis-clinical analysis and novel findings in a series of 20 patients. The Journal of pediatrics 2013;162:850-856.e852.

8. Schmitt SE, Pargeon K, Frechette ES, Hirsch LJ, Dalmau J, Friedman D. Extreme delta brush: a unique EEG pattern in adults with anti-NMDA receptor encephalitis. Neurology 2012;79:1094-1100.

9. Peer M, Pruss H, Ben-Dayan I, Paul F, Arzy S, Finke C. Functional connectivity of largescale brain networks in patients with anti-NMDA receptor encephalitis: an observational study. The lancet Psychiatry 2017;4:768-774.

10. Dale RC, Brilot F, Duffy LV, et al. Utility and safety of rituximab in pediatric autoimmune and inflammatory CNS disease. Neurology 2014;83:142-150.

11. Huberfeld G, Wittner L, Clemenceau S, et al. Perturbed chloride homeostasis and GABAergic signaling in human temporal lobe epilepsy. The Journal of neuroscience : the official journal of the Society for Neuroscience 2007;27:9866-9873.

12. Zwart R, Sher E, Ping X, et al. Perampanel, an antagonist of alpha-amino-3-hydroxy-5methyl-4-isoxazolepropionic acid receptors, for the treatment of epilepsy: studies in human epileptic brain and nonepileptic brain and in rodent models. The Journal of pharmacology and experimental therapeutics 2014;351:124-133.

13. Matricardi S, Patrini M, Freri E, et al. Cognitive and neuropsychological evolution in children with anti-NMDAR encephalitis. J Neurol 2016;263:765-771.

14. Cainelli E, Nosadini M, Sartori S, Suppiej A. Neuropsychological And Psychopathological Profile Of Anti-Nmdar Encephalitis: A Possible Pathophysiological Model For Pediatric Neuropsychiatric Disorders. Archives of clinical neuropsychology : the official journal of the National Academy of Neuropsychologists 2018.

15. Hinkle CD, Porter JN, Waldron EJ, Klein H, Tranel D, Heffelfinger A. Neuropsychological characterization of three adolescent females with anti-NMDA receptor encephalitis in the acute, post-acute, and chronic phases: an inter-institutional case series. The Clinical neuropsychologist 2017;31:268-288. 
16. Anderson V, Spencer-Smith M, Wood A. Do children really recover better?

Neurobehavioural plasticity after early brain insult. Brain : a journal of neurology 2011;134:2197-2221.

17. Petit-Pedrol M, Armangue T, Peng X, et al. Encephalitis with refractory seizures, status epilepticus, and antibodies to the GABAA receptor: a case series, characterisation of the antigen, and analysis of the effects of antibodies. Lancet neurology 2014;13:276-286.

18. Spatola M, Petit-Pedrol M, Simabukuro MM, et al. Investigations in GABAA receptor antibody-associated encephalitis. Neurology 2017;88:1012-1020.

19. Pettingill P, Kramer HB, Coebergh JA, et al. Antibodies to GABAA receptor alpha1 and gamma2 subunits: clinical and serologic characterization. Neurology 2015;84:1233-1241.

20. Doherty J, Quirk M, Piccio L, Zorumski CF.

21. Nikolaus M, Knierim E, Meisel C, et al. Severe GABAA receptor encephalitis without seizures: A paediatric case successfully treated with early immunomodulation. European journal of paediatric neurology : EJPN : official journal of the European Paediatric Neurology Society 2018;22:558-562.

22. O'Connor K, Waters P, Komorowski L, et al. GABAA receptor autoimmunity: A multicenter experience. Neurology(R) neuroimmunology \& neuroinflammation 2019;6:e552. 23. Irani SR, Stagg CJ, Schott JM, et al. Faciobrachial dystonic seizures: the influence of immunotherapy on seizure control and prevention of cognitive impairment in a broadening phenotype. Brain : a journal of neurology 2013;136:3151-3162.

24. Schimmel M, Fruhwald MC, Bien CG. Limbic encephalitis with LGI1 antibodies in a 14year-old boy. European journal of paediatric neurology : EJPN : official journal of the European Paediatric Neurology Society 2018;22:190-193.

25. Hoftberger R, Titulaer MJ, Sabater L, et al. Encephalitis and GABAB receptor antibodies: novel findings in a new case series of 20 patients. Neurology 2013;81:1500-1506.

26. Kruer MC, Hoeftberger R, Lim KY, et al. Aggressive Course in Encephalitis With Opsoclonus, Ataxia, Chorea, and Seizures: The First Pediatric Case of gamma-Aminobutyric Acid Type B Receptor Autoimmunity. JAMA neurology 2014.

27. Laurido-Soto O, Brier MR, Simon LE, McCullough A, Bucelli RC, Day GS. Patient characteristics and outcome associations in AMPA receptor encephalitis. Journal of neurology 2019;266:450-460.

28. Nibber A, Clover L, Pettingill P, et al. Antibodies to AMPA receptors in Rasmussen's encephalitis. European journal of paediatric neurology : EJPN : official journal of the European Paediatric Neurology Society 2016;20:222-227.

29. Carvajal-Gonzalez A, Leite MI, Waters P, et al. Glycine receptor antibodies in PERM and related syndromes: characteristics, clinical features and outcomes. Brain : a journal of neurology 2014;137:2178-2192.

30. Swayne A, Tjoa L, Broadley S, et al. Antiglycine receptor antibody related disease: a case series and literature review. 2018;25:1290-1298.

31. Arino H, Hoftberger R, Gresa-Arribas N, et al. Paraneoplastic Neurological Syndromes and Glutamic Acid Decarboxylase Antibodies. JAMA neurology 2015;72:874-881.

32. Haberlandt E, Bast T, Ebner A, et al. Limbic encephalitis in children and adolescents. Archives of disease in childhood 2011;96:186-191.

33. Pohl D, Alper G, Van Haren K, et al. Acute disseminated encephalomyelitis: Updates on an inflammatory CNS syndrome. Neurology 2016;87:S38-45. 
34. Pohl D, Tenembaum S. Treatment of acute disseminated encephalomyelitis. Current treatment options in neurology 2012;14:264-275.

35. Dale RC. Acute disseminated encephalomyelitis. Seminars in pediatric infectious diseases 2003;14:90-95.

36. Burton KLO, Williams TA, Catchpoole SE, Brunsdon RK. Long-Term

Neuropsychological Outcomes of Childhood Onset Acute Disseminated Encephalomyelitis (ADEM): a Meta-Analysis. Neuropsychology review 2017;27:124-133.

37. Jacobs RK, Anderson VA, Neale JL, Shield LK, Kornberg AJ. Neuropsychological outcome after acute disseminated encephalomyelitis: impact of age at illness onset. Pediatric neurology 2004;31:191-197.

38. Baumann M, Hennes EM, Schanda K, et al. Children with multiphasic disseminated encephalomyelitis and antibodies to the myelin oligodendrocyte glycoprotein (MOG): Extending the spectrum of MOG antibody positive diseases. Multiple sclerosis 2016.

39. Hacohen Y, Wong YY, Lechner C, et al. Disease Course and Treatment Responses in Children With Relapsing Myelin Oligodendrocyte Glycoprotein Antibody-Associated Disease. JAMA Neurol 2018;75:478-487.

40. Ogawa R, Nakashima I, Takahashi T, et al. MOG antibody-positive, benign, unilateral, cerebral cortical encephalitis with epilepsy. Neurology(R) neuroimmunology \& neuroinflammation 2017;4:e322.

41. Hamid SHM, Whittam D, Saviour M, et al. Seizures and Encephalitis in Myelin Oligodendrocyte Glycoprotein IgG Disease vs Aquaporin 4 IgG Disease. JAMA neurology 2018;75:65-71.

42. Rossor T, Benetou C, Wright S, et al. Early predictors of epilepsy and subsequent relapse in children with acute disseminated encephalomyelitis. Multiple sclerosis

2019:1352458518823486.

43. Duignan S, Wright S, Rossor T, et al. Myelin oligodendrocyte glycoprotein and aquaporin-4 antibodies are highly specific in children with acquired demyelinating syndromes. 2018;60:958-962.

44. Zhou J, Lu X, Zhang Y, et al. Follow-up study on Chinese children with relapsing MOGIgG-associated central nervous system demyelination. Multiple sclerosis and related disorders 2019;28:4-10.

45. Ramanathan S, Mohammad S, Tantsis E, et al. Clinical course, therapeutic responses and outcomes in relapsing MOG antibody-associated demyelination. 2018;89:127-137.

46. Balu R, McCracken L, Lancaster E, Graus F, Dalmau J, Titulaer MJ. A score that predicts 1-year functional status in patients with anti-NMDA receptor encephalitis. Neurology 2019;92:e244-e252.

47. Lemon J, Cooper J. Understanding parental perspectives on outcomes following paediatric encephalitis: A qualitative study. 2019;14:e0220042.

48. Mikasova L, De Rossi P, Bouchet D, et al. Disrupted surface cross-talk between NMDA and Ephrin-B2 receptors in anti-NMDA encephalitis. Brain : a journal of neurology 2012;135:1606-1621.

49. Warikoo N, Brunwasser SJ, Benz A, Shu HJ, Paul SM. Positive Allosteric Modulation as a Potential Therapeutic Strategy in Anti-NMDA Receptor Encephalitis. 2018;38:3218-3229.

50. Planaguma J, Haselmann H, Mannara F, et al. Ephrin-B2 prevents N-methyl-D-aspartate receptor antibody effects on memory and neuroplasticity. Annals of neurology 2016;80:388-400. 
51. Finke C, Kopp UA, Scheel M, et al. Functional and structural brain changes in anti-Nmethyl-D-aspartate receptor encephalitis. Annals of neurology 2013;74:284-296.

52. Tewarie P, Schoonheim MM, Stam CJ, et al. Cognitive and clinical dysfunction, altered MEG resting-state networks and thalamic atrophy in multiple sclerosis. PLoS One 2013;8:e69318.

53. Schoonhoven DN, Fraschini M, Tewarie P, et al. Resting-state MEG measurement of functional activation as a biomarker for cognitive decline in MS. Mult Scler 2018:1352458518810260. 